\title{
Intrapulmonary airway smooth muscle is hyperreactive with a distinct proteome in asthma
}

\author{
Gijs ljpma ${ }^{1,2}$, Linda Kachmar ${ }^{1,2}$, Alice Panariti ${ }^{1,2}$, Oleg S. Matusovsky $\mathbb{1}^{3}$, \\ Dara Torgerson ${ }^{2,4}$, Andrea Benedetti, ${ }^{1,6}$ and Anne-Marie Lauzon ${ }^{1,2}$
}

Affiliations: 'Dept of Medicine, McGill University, Montreal, QC, Canada. ${ }^{2}$ Meakins-Christie Laboratories, Research Institute of the McGill University Health Centre, Montreal, QC, Canada. ${ }^{3}$ Dept of Kinesiology and Physical Education, McGill University, Montreal, QC, Canada. ${ }^{4}$ McGill University and Génome Québec Innovation Centre, Montreal, QC, Canada. ${ }^{5}$ Dept of Epidemiology, Biostatistics and Occupational Health, McGill University, Montreal, QC, Canada. ${ }^{6}$ Respiratory Epidemiology and Clinical Research Unit, McGill University Health Centre, Montreal, QC, Canada.

Correspondence: Anne-Marie Lauzon, 1001 Decarie Blvd. EM3.2236, Montreal, QC, H4A3J1, Canada. E-mail: anne-marie.lauzonamcgill.ca

@ERSpublications

Intrapulmonary, but not tracheal, airway smooth muscle is hyperreactive in asthma, together with pro-contractile changes in the airway smooth muscle proteome. Several proteins were identified that could be targeted for treatment of the hyperreactivity. http://bit.ly/33esYSU

Cite this article as: Ijpma G, Kachmar L, Panariti A, et al. Intrapulmonary airway smooth muscle is hyperreactive with a distinct proteome in asthma. Eur Respir J 2020; 56: 1902178 [https://doi.org/10.1183/ 13993003.02178-2019].

ABSTRACT Constriction of airways during asthmatic exacerbation is the result of airway smooth muscle (ASM) contraction. Although it is generally accepted that ASM is hypercontractile in asthma, this has not been unambiguously demonstrated. Whether airway hyperresponsiveness (AHR) is the result of increased ASM mass alone or also increased contractile force generation per unit of muscle directly determines the potential avenues for treatment.

To assess whether ASM is hypercontractile we performed a series of mechanics measurements on isolated ASM from intrapulmonary airways and trachealis from human lungs. We analysed the ASM and whole airway proteomes to verify if proteomic shifts contribute to changes in ASM properties.

We report an increase in isolated ASM contractile stress and stiffness specific to asthmatic human intrapulmonary bronchi, the site of increased airway resistance in asthma. Other contractile parameters were not altered. Principal component analysis (PCA) of unbiased mass spectrometry data showed clear clustering of asthmatic subjects with respect to ASM specific proteins. The whole airway proteome showed upregulation of structural proteins. We did not find any evidence for a difference in the regulation of myosin activity in the asthmatic ASM.

In conclusion, we showed that ASM is indeed hyperreactive at the level of intrapulmonary airways in asthma. We identified several proteins that are upregulated in asthma that could contribute to hyperreactivity. Our data also suggest enhanced force transmission associated with enrichment of structural proteins in the whole airway. These findings may lead to novel directions for treatment development in asthma.

All analysed data and materials associated with this study are in the paper, raw data are available upon request.

This article has supplementary material available from erj.ersjournals.com

Received: 8 Nov 2019 | Accepted after revision: 5 March 2020

Copyright $\odot$ ERS 2020 


\section{Introduction}

Asthma, a common chronic disease affecting over 300 million people worldwide [1], is characterised by exaggerated constriction of the airways (i.e. airway hyperresponsiveness (AHR)) in response to environmental stimuli. The causes of asthma are still unknown and the mechanisms underlying excessive bronchoconstriction are poorly characterised. In particular, while the role of airway smooth muscle (ASM) in bronchoconstriction is undeniable, no study has unambiguously shown hypercontractility of asthmatic ASM. A major problem with previous measurements is that they were either performed on isolated trachealis ASM, a tissue that is clinically irrelevant for asthma studies, or on whole intrapulmonary airway rings or strips, without isolating the muscle per se. In developing novel treatments for asthma, understanding whether or not we're up against hypercontractile ASM will be an essential part of the puzzle.

We and others have previously shown that the contractile properties of human trachealis and main bronchi smooth muscle are not intrinsically altered in asthma [2, 3], although CHIN et al. [3] did find a difference in the response to large length oscillations. However, we have subsequently shown, in the horse model of spontaneously occurring asthma, that intrapulmonary (IP) ASM exhibits a significant increase in the maximal shortening velocity $\left(\mathrm{V}_{\max }\right.$, the maximum rate of shortening) but that trachea $(\mathrm{T})$ does not [4]. This increase was dependent on the time since the last steroid treatment, suggesting that it resulted from the inflammatory environment of the IP ASM [5]. In human asthma, inflammation has been shown to progressively increase towards the periphery [6], which may result in site-specific ASM behaviour. Indeed, by exposing rat trachealis smooth muscle to inflammatory cells, we were able to recreate the increased $\mathrm{V}_{\max }$ observed in the horse IP ASM [7]. Furthermore, in response to a single dose of anti-inflammatory drugs, subjects with asthma show a rapid but transient reduction in AHR [8, 9]. While reduced mucous content and airway swelling likely contribute to this [10], our studies suggest that a transient change in the ASM mechanics may also contribute to the reduced AHR.

Thus, to assess whether IP ASM itself exhibits greater contractility in asthma, we isolated IP (3rd to 5th branching generation) and trachealis ASM strips from fresh transplant-grade lungs from subjects with asthma and controls, and compared their contractile properties. We subsequently performed mass spectrometry measurements on the IP airways to investigate the observed contractility changes.

\section{Materials and methods}

\section{Procurement and tissue preparation}

Fresh asthmatic and control transplant-grade lungs were procured by the International Institute for the Advancement of Medicine and the National Disease Research Interchange. The study was approved by the McGill University Health Center Research Ethics Board. Criteria for inclusion as asthmatic were based on either existing medical records of asthma diagnosis where available, or on next-of-kin reporting of an asthma diagnosis. Detailed preparation and transport protocols are described in the supplementary material. Demographics and summaries of donor clinical details are shown in table 1, with further details in supplementary table S1.

Upon arrival, lung lobes were separated and the lower right and left lobes were placed in oxygenated Hank's balanced salt solution at $4{ }^{\circ} \mathrm{C}$ for initial dissection (solution compositions are given in the supplementary material), while remaining lobes were used for other studies. Trachealis strips were isolated as previously described [2]. For ASM dissection details see the supplementary material.

\section{Mechanics measurements}

Muscle tissue strips were suspended in a horizontal tissue bath as previously described [2]. In cases where the in situ length could not be determined, the tissues were stretched to just above slack length [11]. Initial tests in our lab and in other studies $[12,13]$, have shown that after an equilibration period with repeated contractions, smooth muscle contractile stress and $\mathrm{V}_{\max }$ (in current lengths $\cdot \mathrm{s}^{-1}$ ) is independent of muscle

TABLE 1 Subject summary table and number of subjects assessed for each dataset

\begin{tabular}{|c|c|c|c|c|c|c|c|c|c|}
\hline & Subjects & Sex $(F / M)$ & Age years & BMI $\mathrm{kg} \cdot \mathrm{m}^{-2}$ & \multicolumn{4}{|c|}{ Mechanics (\#IB - \#T) } & Proteomics \\
\hline Asthma & 12 & $5 / 7$ & $39 \pm 4$ & $29 \pm 2$ & $5-8$ & $4-5$ & $7-9$ & $6-7$ & 6 \\
\hline Control & 19 & $8 / 11$ & $43 \pm 3$ & $28 \pm 1$ & $7-11$ & $4-4$ & $10-13$ & $7-10$ & 6 \\
\hline
\end{tabular}

Data is presented as $n, n / n$, mean \pm SEM. \#IB - \#T is the number of subjects for intrapulmonary bronchi versus trachealis airway smooth muscle (ASM). BMI: body mass index; MCh: methacholine; Iso: isoproterenol; $\mathrm{V}_{\text {max }}$ : maximal shortening velocity. 
length for a wide range of muscle lengths. The starting length will be referred to as the reference length $\left(\mathrm{L}_{\text {ref }}\right)$. Tissues were continuously flushed with Krebs solution at $1 \mathrm{~mL} \cdot \mathrm{min}^{-1}$. For detailed description of equilibration and mechanics protocols, see the supplementary material. In short, after an $\sim 1 \mathrm{~h}$ equilibration period, we performed shortening velocity measurements using repeated isotonic contractions (see supplementary figure S1b). A single methacholine (MCh) $10^{-5} \mathrm{M}$ contraction with superimposed length oscillations, small enough to not affect the average contractile stress, was used to measure the viscoelastic properties of the tissues (see supplementary figure S1c). Lastly, we performed MCh and isoproterenol (Iso) dose-response measurements (see supplementary figure S1a). ASM area derived from histology of the smooth muscle strip was used to calculate contractile stress from maximal force response values.

To reduce variability and in case of data rejection (supplementary rejection criteria), we tested two IP and two trachealis tissues for each lung. For details see supplementary table S1.

\section{Mass spectrometry}

Ultra high performance liquid chromatography-tandem mass spectrometry (UHPLC-MS/MS) (Dionex Ultimate 3000) was used on extracts from whole airways with parameters as described in the supplementary material. Smooth muscle specific proteins were identified according to The Protein Atlas [14] (see the supplementary material). Spectrum counts were normalised to total smooth muscle content using the sum of relative total exclusive spectrum counts for each smooth muscle protein.

\section{Statistics}

We used linear mixed models to estimate the difference for all mechanics measurements, with a random intercept to account for measurements taken from the same subject ( $\mathrm{n}$ is number of subjects tested). Means, standard errors and p-values were calculated from the mixed model regression analyses. Error bars are standard errors, geometric means are shown for $\mathrm{EC}_{50}$ data (where $\mathrm{EC}_{50}$ is the dose at which $50 \%$ of maximal stress is generated). Principal component analysis (PCA) on proteomics data was performed in Matlab $^{\text {TM }}$ (version R2018b, Mathworks). Significance of clustering of asthmatic patients and controls was tested by two-sided unpaired T-tests on the scores along the first principal component (PC1). Tests for functional enrichment of proteins that varied in levels between asthmatic patients and controls were performed using the Database for Annotation, Visualisation and Integrated Discovery (DAVID), version 7.6 [15], with Bonferroni correction.

\section{Results}

\section{Methacholine and isoproterenol dose-response measurements}

In the MCh dose-response measurements for isolated ASM strips from IP airways, we observed a markedly increased maximal stress $\left(\sigma_{\max }\right)$, as derived from dose-response curve fits to data (figure 1a), in asthmatic patients compared to control patients ( $104.9 \pm 13.1 \mathrm{kPa}$ versus $56.5 \pm 6.7 \mathrm{kPa} ; \mathrm{p}=0.04$ ) (figure $1 \mathrm{~b}$ ); however, this difference was not observed in trachealis ASM $(95.8 \pm 14.2 \mathrm{kPa}$ versus $109.5 \pm 14.8 \mathrm{kPa}$; $=0.46)$ (figure $1 \mathrm{~b})$. A significant interaction $(\mathrm{p}<0.05)$ between disease state and site was found, indicating that the IP and trachealis ASM were affected differently by the disease. Asthmatic IP ASM was found to be hyposensitive compared to controls (geometric means of $\mathrm{EC}_{50}: 5.6 \pm 0.12-\log _{10}(\mathrm{M} \mathrm{MCh})$ versus $6.1 \pm 0.14-$ $\log _{10}(\mathrm{M} \mathrm{MCh}$ ); $\mathrm{p}=0.02$ ) (figures $1 \mathrm{c}$ and $1 \mathrm{~d}$ ), whereas trachealis ASM did not show any significant difference (geometric means: $5.9 \pm 0.14-\log _{10}(\mathrm{M} \mathrm{MCh})$ versus $\left.6.2 \pm 0.15-\log _{10}(\mathrm{M} \mathrm{MCh}) ; \mathrm{p}=0.17\right)$.

We found no differences in the maximum relaxation to Iso in IP ASM (asthma versus control: $61.2 \pm 12.9 \%$ versus $66.2 \pm 4.8 \% ; \mathrm{p}=0.73$ ) or trachealis ASM (asthma versus control: $58.3 \pm 12.3 \%$ versus $59.5 \pm 3.9 \%$; $\mathrm{p}=0.92$ ) (figures $1 \mathrm{e}$ and $1 \mathrm{f})$ and no significant interaction $(\mathrm{p}=0.75)$. Likewise, we found no significant difference in Iso $\mathrm{EC}_{50}$ geometric means for asthma versus control (IP) $\left(7.00 \pm 0.35-\log _{10}\right.$ (M Iso) versus $7.09 \pm 0.28-\log _{10}$ (M Iso); $\left.\mathrm{p}=0.84\right)$ or for asthma versus control (trachealis) $\left(6.81 \pm 0.33-\log _{10}\right.$ (M Iso) versus $6.72 \pm 0.19-\log _{10}$ (M Iso); $\mathrm{p}=0.84$ ) (figure $1 \mathrm{~g}$ ), with no significant interaction between disease and location.

\section{Maximal shortening velocity and viscoelastic properties}

To assess whether $V_{\max }$ is changed in human asthma and whether these changes are specific to different phases of contraction, we calculated $\mathrm{V}_{\max }$ during electrical field stimulation (EFS) at three timepoints $(5 \mathrm{~s}, 8 \mathrm{~s}$ and $10 \mathrm{~s}$ after the initiation of contraction) by extrapolating from force-velocity curves (figures $2 \mathrm{a}-2 \mathrm{c}$ ). We found no significant differences in $\mathrm{V}_{\max }$ between asthma patients and controls and no time effect. We did find a significant effect of location on $\mathrm{V}_{\max }(\mathrm{p}<0.001)$ (figure $2 \mathrm{~d}$ ).

To probe the viscoelastic properties of the muscle, we applied continuous small sinusoidal length oscillations for the duration of a single MCh contraction. Mean stress (figures $2 \mathrm{e}$ and $2 \mathrm{f}$ ) and stiffness (figures $2 \mathrm{~g}$ and 2h) followed similar trajectories during contraction in trachealis ASM and IP ASM. A greater mean stress 

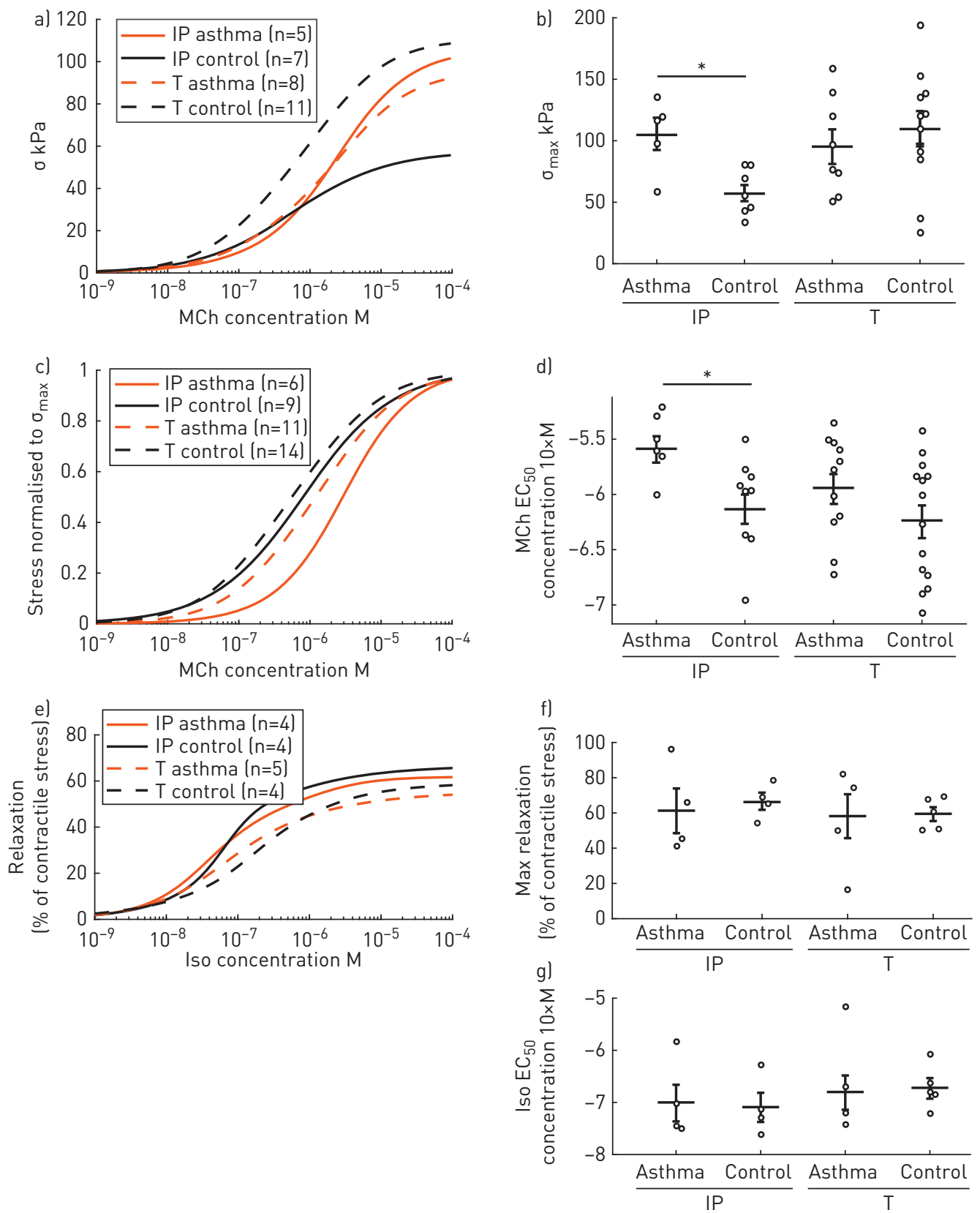

FIGURE 1 Dose responses to methacholine (MCh) and isoproterenol (Iso). Black lines: controls; orange lines: subjects with asthma; solid lines: intrapulmonary (IP); dashed lines: trachea (T). Panels are as follows: a) absolute MCh dose-response curves (stress ( $\sigma$ ) versus MCh concentration); b) dot plot of maximal stress $\left(\sigma_{\text {max }}\right)$; c) normalised MCh dose-response curves (normalised stress versus MCh concentration); d) dot plot of sensitivity to $\mathrm{MCh}$ as expressed by $\mathrm{EC}_{50}$ (dose at which $50 \%$ of maximal stress is generated); e) Iso doseresponse curve with relaxation expressed as a percentage of the maximal contractile stress; $f$ ) dot plot of maximum relaxation; $g$ ) dot plot of Iso sensitivity as expressed by $\mathrm{EC}_{50}$. $^{*}$ : $\mathrm{p}<0.05$.

$(142 \pm 47 \mathrm{KPa}$ versus $52.7 \pm 5.7 \mathrm{KPa} ; \mathrm{p}=0.047)$ and stiffness $(10 \pm 2.7 \mathrm{MPa}$ versus $3.7 \pm 5.2 \mathrm{MPa} ; \mathrm{p}=0.02)$ were observed in contracted asthmatic IP ASM compared to controls, with no differences in trachealis ASM. No significant difference was found in the baseline values (i.e. relaxed ASM stress and stiffness). Our data on the viscous properties of the tissues, as expressed by the dimensionless parameter hysteresivity (figures $2 \mathrm{i}$ and $2 \mathrm{j}$ ), showed no differences between trachealis or peripheral ASM from subjects with asthma or from controls.

\section{Protein expression}

To narrow down the possible causes of the hyperreactivity, we performed unbiased mass spectrometry on whole airway samples from the same locations as our IP mechanics samples (see supplementary table S3). 

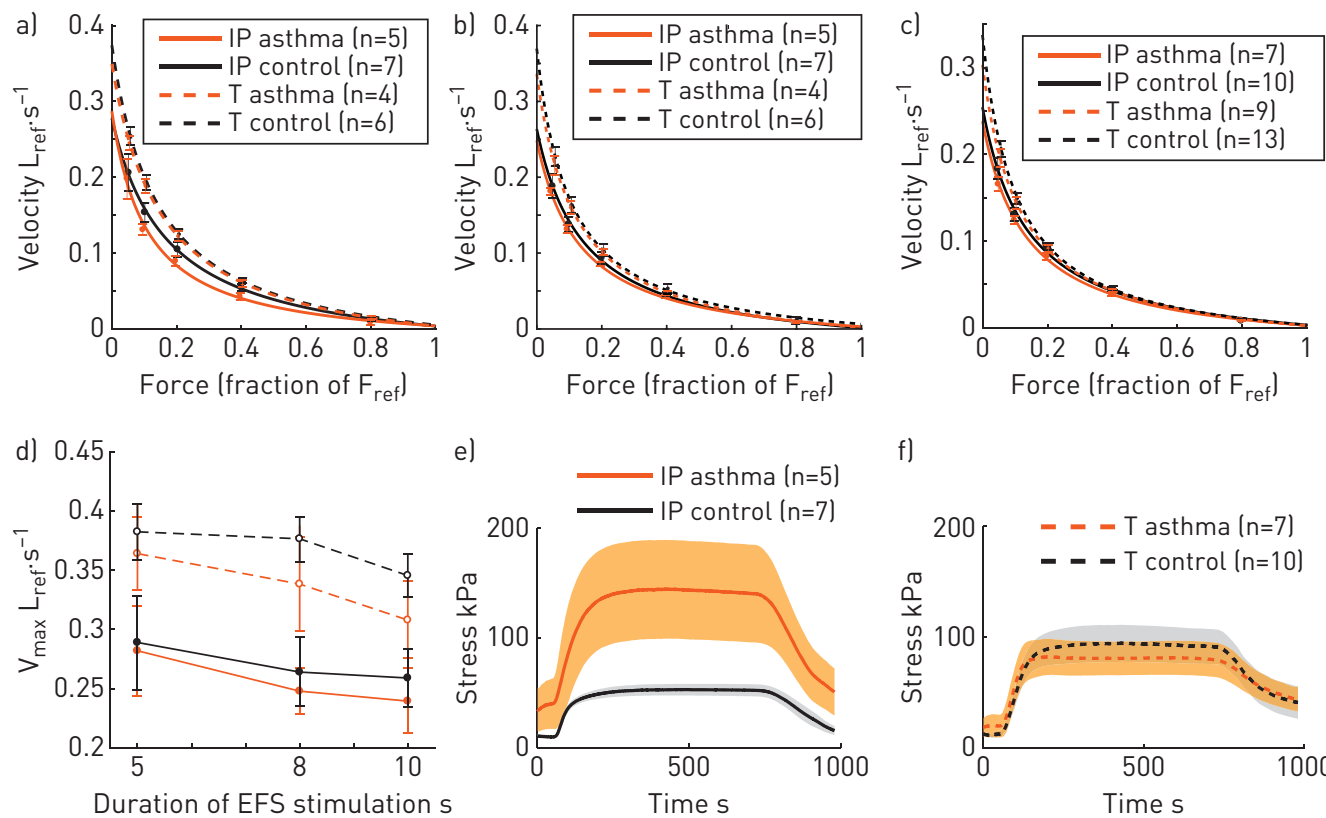

f)
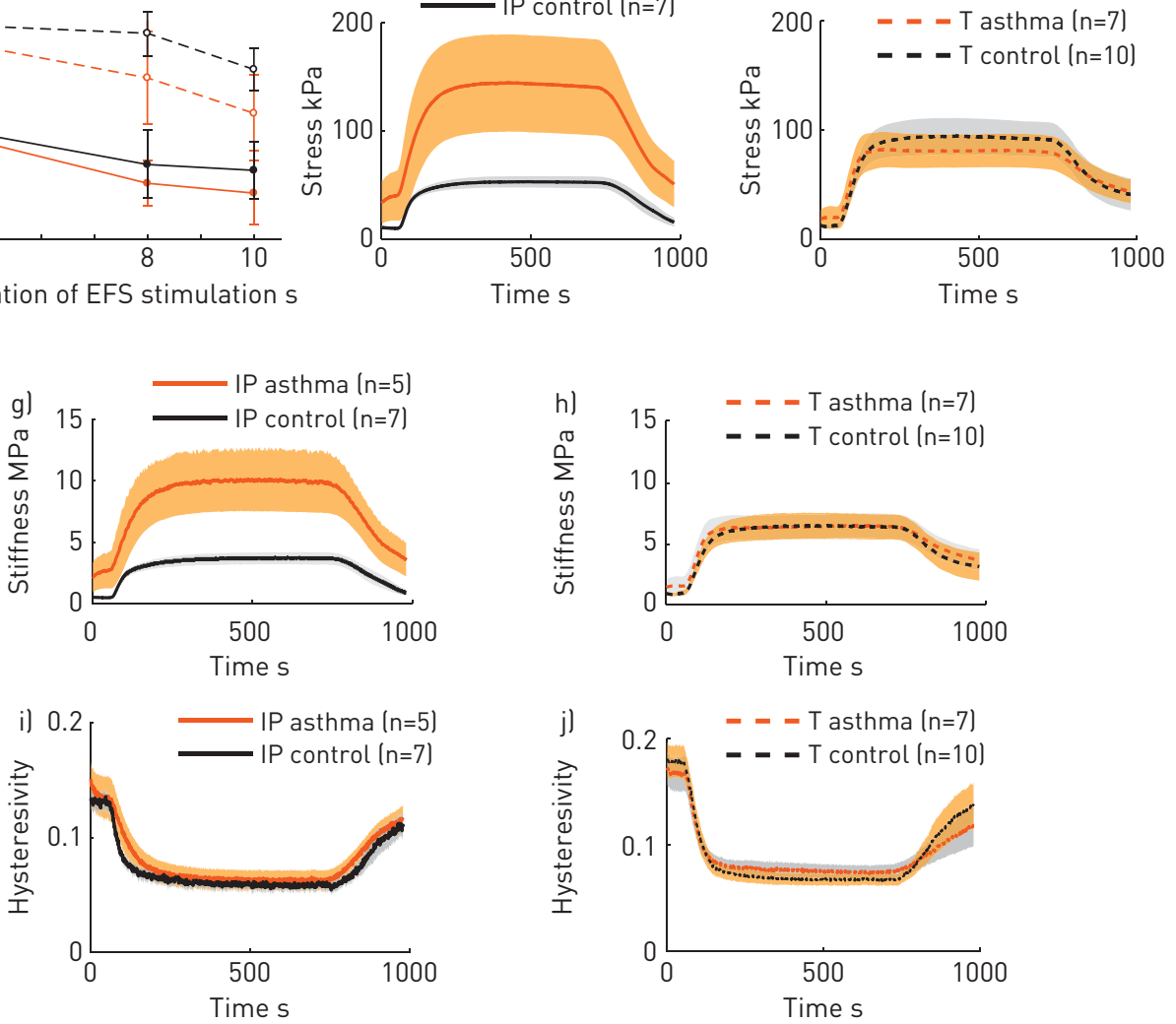

FIGURE 2 Force-velocity curves during electrical field stimulation (EFS) and stress, stiffness and hysteresivity during methacholine ( $\mathrm{MCh}$ ) contraction. Black lines: controls; orange lines: subjects with asthma; solid lines: intrapulmonary (IP); dashed lines: trachea (T). Panels are as follows: al force-velocity curves at $5 \mathrm{~s}$ into EFS; b) force-velocity curves at $8 \mathrm{~s}$ into EFS; c) force-velocity curves at $10 \mathrm{~s}$ into EFS; d) maximal shortening velocity ( $V_{\max }$ ) from $\left.a-c ; e\right)$ and $f$ ) mean stress as a function of time; $g$ ) and h) stiffness normalised to length as a function of time; i) and j) hysteresivity obtained by length oscillations during MCh stimulation as a function of time. Light gray and orange areas: $95 \%$ confidence interval $(\mathrm{Cl})$. $\mathrm{L}_{\text {ref: }}$ reference length; $\mathrm{F}_{\mathrm{ref}}$ : contractile force just prior to isotonic contraction.

To assess smooth muscle specific changes, we included only those proteins that are specific to smooth muscle ( 42 out of 2272 detected proteins) (see supplementary table S2 and supplementary figure S2a). The data heatmap of these proteins shows separation of asthmatic and control subjects (figure 3a), except for subject 28 (control) and, to a lesser extent, subject 12 (asthma). As subject 28 may be a clinical outlier amongst controls because of the combination of high body mass index (BMI), extensive home medication and potentially strong inflammation associated with autism [16], this subject was excluded from further analyses. PCA on the proteomics data showed significant separation of asthma patients and controls along $\mathrm{PC1}$, with $42 \%$ of variance explained (scores $1.19 \pm 1.34$ versus $-1.43 \pm 0.45 ; \mathrm{p}=0.0025$ ) (figure $3 \mathrm{~b}$ ). For inclusion of subject 28 see supplemental figure S2b. In PCA of the total detected proteome of the airway samples (see supplemental figure S3a), asthmatic subjects and control subjects do not show significant separation along the two PCs, with or without subject 28 (supplemental figure S3b and figure 3d, respectively). 

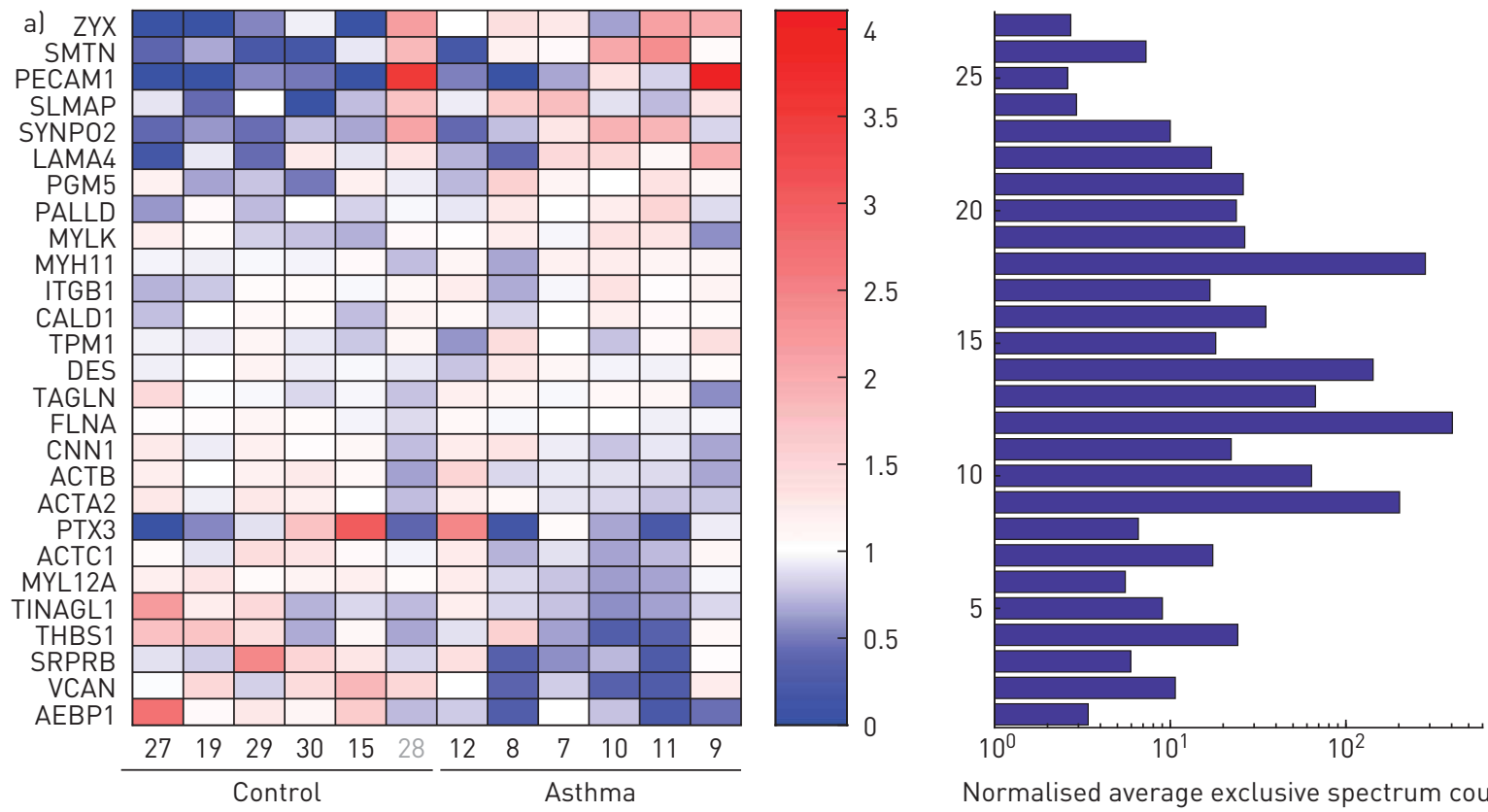

Normalised average exclusive spectrum count
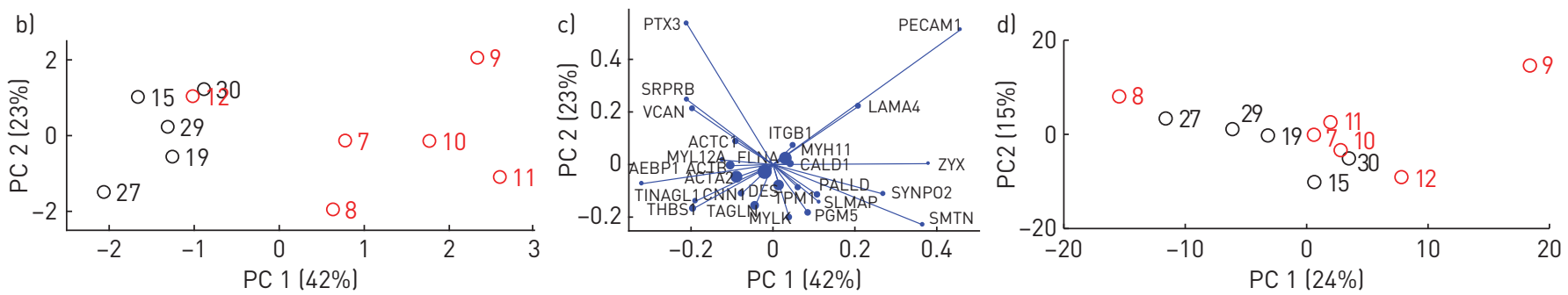

FIGURE 3 Proteomics analysis from airways of asthmatic and control subjects. Panels are as follows: a) heatmap of relative protein exclusive spectrum counts (left) with averaged normalised exclusive spectrum counts across all subjects for each protein (right); b) plot of the first two principal components (PC1 and PC2) for all subjects, except the clinical outlier subject 28, for smooth muscle specific proteins only. Black open circles: control subjects; red open circles: asthmatic subjects; c) contribution of each protein to PC1 and PC2 for smooth muscle specific proteins only; d) plot of PC1 and PC2 for all subjects, except the clinical outlier subject 28, for whole airway proteins. Black open circles: control subjects; red open circles: asthmatic subjects.

Effects of body mass index, age and gender

We tested the correlation of $\sigma_{\max }, \mathrm{MCh}$ dose-response $\mathrm{EC}_{50}$ and $\mathrm{V}_{\max }$ with subject $\mathrm{BMI}$, age and gender. With Bonferroni correction for multiple correlation testing we found no significant partial correlations between the contractility parameters and each of the subject characteristics while controlling for the remaining two subject characteristics (figure 4).

\section{Discussion}

Airway constriction in asthma has long been associated, mostly intuitively, with hyperreactivity (greater maximal response) and hypersensitivity (greater response at low doses) of ASM to contractile agonists. Previous attempts to verify this in isolated ASM tissues showed no differences between control and asthmatic trachealis [2,3] or main bronchi ASM [2]. Earlier studies looked at contractility in asthma of airway strips with epithelium [17-24], which is known to modulate the behaviour of ASM [25], or whole airway rings [22, 23], but no isolated IP ASM tissues were examined. Furthermore, most of these tissues were taken from lung resections of heavy smokers $[17,18,22,23]$ or were taken many hours post-mortem [19-21]. Whole airway or airway strip contractile force was not changed in asthma [17-21], but increased when normalised for ASM cross-sectional area [22]. Increased isolated airway narrowing was also found in subjects with asthma [23]. In our study we measured the contractility of isolated IP ASM and extrapulmonary ASM. We showed that hyperreactivity of ASM is a feature of asthma after all, but that it is limited to the site of asthmatic attacks (the IP bronchi).

It is worth noting that the contractile stress in our IP ASM from control subjects is lower compared to all tracheal ASM tissues and asthmatic IP ASM tissues (figure 1b). Our contractile stress values correspond 

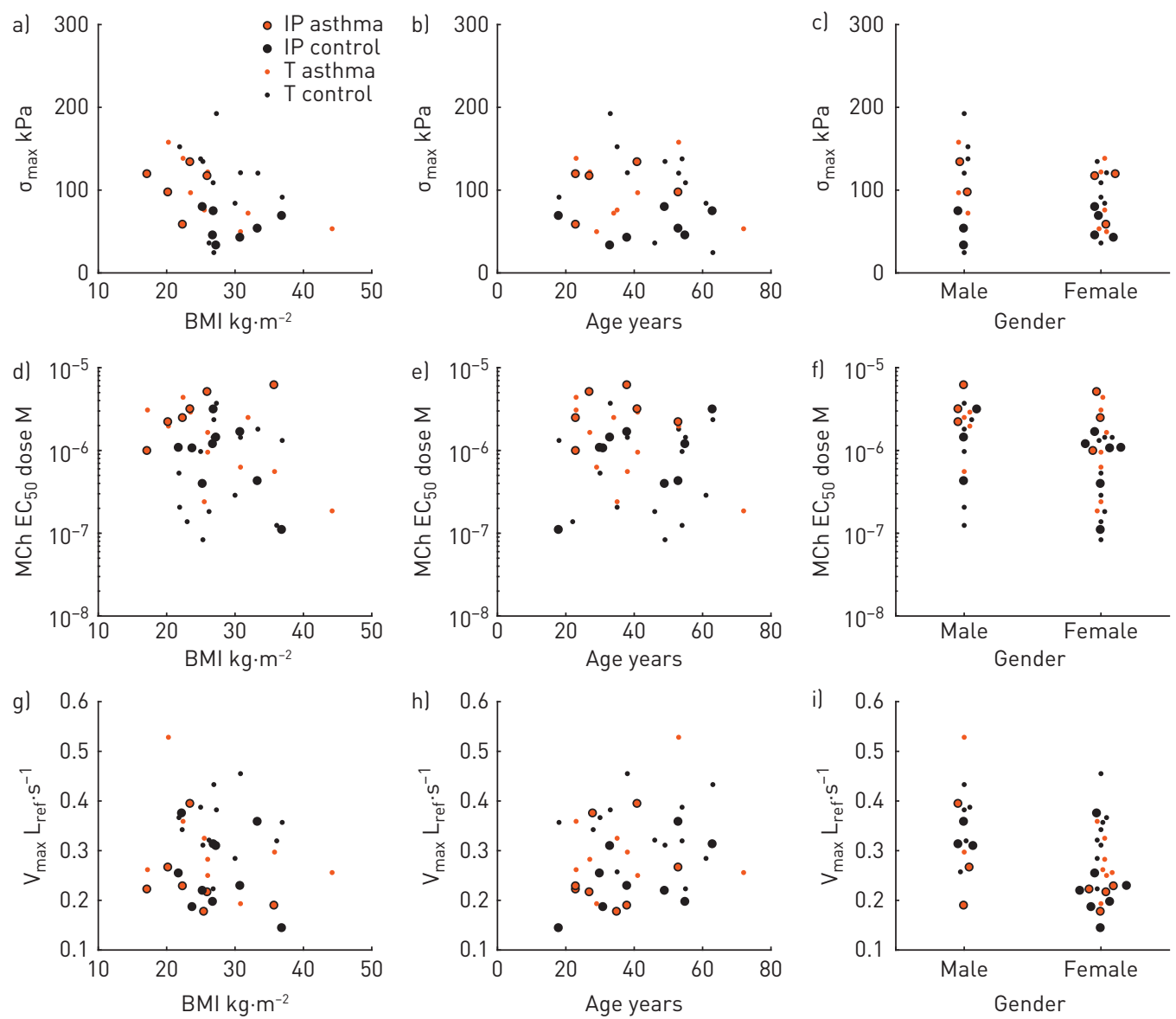

FIGURE 4 Correlations of body mass index (BMI), age and sex for three contractility parameters: maximal stress $\left(\sigma_{\max }\right)(a, b, c)$, methacholine $(\mathrm{MCh}) \mathrm{EC}_{50}$ dose (dose at which $50 \%$ of maximal stress is generated) $(d, e, f)$ and maximal shortening velocity $\left(V_{\max }\right)(g, h, i)$.

with published values from the same locations (ASM in healthy whole human airways [22] and trachealis ASM from healthy and asthmatic subjects) [3]. This difference in healthy IP ASM is possibly the result of a difference in function. In the healthy lung, the function of ASM in IP bronchi is unknown (if there is a function at all) [26], while trachealis ASM is activated during cough [27] to aid in expelling foreign objects. This difference in function may lead to suboptimal adaptation for force generation in healthy IP ASM, which may be changed by inflammatory stimuli in asthma. Site specificity of the inflammatory environment may drive site specific changes in contractile properties and several studies have shown progressively more severe inflammation towards the periphery $[5,6,28]$. Our previous studies in animal models of asthma support the role of inflammatory cells and mediators in altering ASM contractile properties [4, 7], as well as their partial reversal being induced by corticosteroids [4]. A recent study showed site specificity in rho kinase 1 and rho kinase 2 content in ASM, with higher concentrations in the asthmatic IP airways [29] potentially resulting from inflammation. Rho kinase inhibitors have been shown to reduce ASM contractile stress and resistance to length oscillations [30]. It was also recently shown that inflammation may directly modulate ASM contractility through upregulation and activation of the calcium sensing receptor [31]. The full effects of inflammatory cells on human ASM mechanical properties will require further investigation.

The paradoxically small change in MCh sensitivity, which mirrors the change we previously showed in main bronchi smooth muscle [2], is likely clinically irrelevant. It may indicate that sensitivity is primarily caused by changes in the epithelial barrier function in asthma [32-34], which can modulate the sensitivity to contractile agonists by up to three orders of magnitude [25]. Human asthmatic bronchial strip preparations have previously shown either hyposensitivity to contractile agonists in asthmatic subjects [19, 20], in agreement with the current study, or no change [21,24]. In three of these studies, Iso showed a decrease in sensitivity in both fatal $[19,21]$ and non-fatal [24] asthma, but a study on mostly mild cases found no change [20]. Our data, which are mostly from mild to moderate asthma subjects, seem to agree with this latter study. 
Based on prior studies on horse IP ASM [4] and co-culture of rat trachealis with T-cells [7], we had hypothesised that $\mathrm{V}_{\max }$ in human IP ASM would also be increased. However, we did not find any changes in $\mathrm{V}_{\max }$ at either location in asthma, or any time dependence despite the large change in contractile stress over this time range. Future studies into time dependence of $\mathrm{V}_{\max }$ in longer, agonist induced contractions may yield different data. Alternatively, the lack of difference in $\mathrm{V}_{\max }$ between control subjects and asthma subjects may lie in the type of asthma. In the horse model of spontaneously occurring asthma [4], all horses are considered severe cases and their inflammation is primarily neutrophilic compared to eosinophilic in humans. These horses, as well as induced animal models of asthma, may have a much more persistent inflammatory environment in the lung than the mild to moderate asthmatics in the current study. In fact, we previously showed that rat trachealis exposed for only $24 \mathrm{~h}$ to $\mathrm{CD}_{4}^{+} \mathrm{T}$-cells had an increased $\mathrm{V}_{\max }$ [7], suggesting that $\mathrm{V}_{\max }$ is increased only during exacerbations. Changes in stress may require challenges to occur over a much longer period, which may explain their absence in most animal models. Our data on viscoelastic properties indicate that these more persistent changes in contractile stress do not affect the viscous properties of the muscle. If the viscous properties of cells are the result of frictional forces between proteins [35], the lack of change in viscosity paired with an increase in contractile stress and stiffness thus point to increased force generation and/or force transmission without increased overall protein size and density.

Regardless of the mechanism responsible for the enhanced contractility found in asthmatic IP ASM, the proteomic signature of ASM gives a snapshot of the contractile apparatus and structural proteins that existing studies on body fluid proteomics [36], genome wide association studies [37] and RNA expression studies [38] cannot address. To date this is the first study to address and find proteomic differences of ASM tissues between subjects with asthma and control subjects. Despite a relatively small sample size, a clear separation between asthmatic patients and control patients was found in PCA, indicating that the relative protein composition of asthmatic ASM is indeed different from controls. Among the proteins that contribute most to PC1 (figure 3c), increased zyxin (ZYX) [39] and smoothelin (SMTN) [38] have previously been implicated in asthma. ZYX facilitates contractile recovery from stretch, such as occurs during deep inspiration, by repairing fragmented stress fibres [39]. Increased SMTN has been associated with contractile smooth muscle (in contrast to proliferative smooth muscle) [40], while the inverse was found for decreased adipocyte enhancer-binding protein 1 (AEBP1) [41]. Increased synaptopodin-2 (SYNPO2) is linked to an increased filamentous to globular actin ratio [42].

PCA of the whole airway proteome (see supplementary figure S3a) did not show clear clustering of asthmatic and control subjects, with or without subject 28 (see supplementary figure S3b and figure $3 \mathrm{~d}$, respectively). We tested for functional enrichment in proteins that varied between subjects with asthma and control subjects using DAVID [15], with total discovered proteins as background. All proteins (66) were increased in asthma subjects (see supplementary figure S3c). Cell component gene ontology (see supplementary table S4) showed enrichment of "cytoskeleton" and its subset "actin cytoskeleton", as well as "focal adhesions" and "adherens junctions". These results show that these structural proteins are increased relative to total airway wall protein content, which may reflect increased structural integrity and force transmission in the airway wall.

Asthma is fundamentally a mechanical disease with a poorly understood aetiology, but which undoubtedly results in excessive airway constriction. Our study showed new evidence for the role of ASM in airway hyperresponsiveness, clearly demonstrating that airway origin matters when it comes to ASM contractility. We identified several proteins that require further study to understand their role in ASM hyperreactivity in asthma and which may lead to avenues for treatment.

Acknowledgements: We thank the proteomics core facility of the Research Institute of the McGill University Health Centre for their help with acquiring the mass spectrometry data. We also thank James G. Martin (Dept of Medicine, McGill University, Montreal, QC, Canada) for assistance in developing recovery protocols for donor lungs and interpretation of medical data.

Author contributions: G. Ijpma: conception and design; acquisition of data; analysis and interpretation of data; drafting and review of manuscript; L. Kachmar: acquisition of data; article review; A. Panariti: acquisition of data; analysis of data; article review; O.S. Matusovsky: acquisition of data; article review; D. Torgerson: proteomics analysis advice; article review; A. Benedetti: statistical advice; article review; A-M. Lauzon: conception and design; analysis and interpretation of data; drafting and review of manuscript.

Conflict of interest: None declared.

Support statement: This work was supported by a National Heart, Lung and Blood Institute grant (RO1-HL 103405-02), the Canadian Institute for Health Research and the Costello Fund. The Meakins-Christie Laboratories of the Research Institute of McGill University Health Center are supported in part by a centre grant from the Fonds de la Recherche en Santé Respiratoire du Québec. Funding information for this article has been deposited with the Crossref Funder Registry. 


\section{References}

$1 \quad$ Papi A, Brightling C, Pedersen SE, et al. Asthma. Lancet 2018; 391: 783-800.

2 Ijpma G, Kachmar L, Matusovsky OS, et al. Human trachealis and main bronchi smooth muscle are normoresponsive in asthma. Am J Respir Crit Care Med 2015; 191: 884-893.

3 Chin LYM, Bossé Y, Pascoe C, et al. Mechanical properties of asthmatic airway smooth muscle. Eur Respir J 2012; 40: $45-54$.

4 Matusovsky OS, Kachmar L, Ijpma G, et al. Peripheral airway smooth muscle but not the trachealis is hypercontractile in an equine model of asthma. Am J Respir Cell Mol Biol 2015; 54: 718-727.

5 Hamid Q, Song Y, Kotsimbos TC, et al. Inflammation of small airways in asthma. J Allergy Clin Immunol 1997; 100: 44-51.

6 De Magalhães Simões S, Dos Santos MA, Da Silva Oliveira M, et al. Inflammatory cell mapping of the respiratory tract in fatal asthma. Clin Exp Allergy 2005; 35: 602-611.

7 Matusovsky OS, Nakada EM, Kachmar L, et al. CD4 ${ }^{+} \mathrm{T}$ cells enhance the unloaded shortening velocity of airway smooth muscle by altering the contractile protein expression. J Physiol 2014; 592: 2999-3012.

8 Berge MVD, Luijk B, Bareille P, et al. Prolonged protection of the new inhaled corticosteroid fluticasone furoate against AMP hyperresponsiveness in patients with asthma. Allergy 2010; 65: 1531-1535.

9 Luijk B, Kempsford RD, Wright AM, et al. Duration of effect of single-dose inhaled fluticasone propionate on AMP-induced bronchoconstriction. Eur Respir J 2004; 23: 559-564.

10 James A, Carroll N. Theoretic effects of Mucus Gland Discharge on airway resistance in asthma. Chest 1995; 107: Suppl. 3, 110S.

11 Bates JHT, Bullimore SR, Politi AZ, et al. Transient oscillatory force-length behavior of activated airway smooth muscle. Am J Physiol Lung Cell Mol Physiol 2009; 297: L362-L372.

12 Pratusevich VR, Seow CY, Ford LE. Plasticity in canine airway smooth muscle. J Gen Physiol 1995; $105:$ 73-94.

13 Smolensky AV, Ford LE. The extensive length-force relationship of porcine airway smooth muscle. J Appl Physiol 2007; 102: 1906-1911.

14 Uhlén M, Fagerberg L, Hallström BM, et al. Tissue-based map of the human proteome. Science 2015; 347: 1260419 .

15 Huang DW, Sherman BT, Lempicki RA. Systematic and integrative analysis of large gene lists using DAVID bioinformatics resources. Nature Protocols 2008; 4: 44.

16 Siniscalco D, Schultz S, Brigida AL, et al. Inflammation and neuro-immune dysregulations in autism spectrum disorders. Pharmaceuticals (Basel) 2018; 11: 56.

17 de Jongste JC, van Strik R, Bonta IL, et al. Measurement of human small airway smooth muscle function in vitro with the bronchiolar strip preparation. J Pharmacol Methods 1985; 14: 111-118.

18 Ishida K, Pare PD, Hards J, et al. Mechanical properties of human bronchial smooth muscle in vitro. J Appl Physiol 1992; 73: 1481-1485.

19 Goldie RG, Spina D, Henry PJ, et al. In vitro responsiveness of human asthmatic bronchus to carbachol, histamine, beta-adrenoceptor agonists and theophylline. Br J Clin Pharmacol 1986; 22: 669-676.

20 Whicker SD, Armour CL, Black JL. Responsiveness of bronchial smooth muscle from asthmatic patients to relaxant and contractile agonists. Pulm Pharmacol 1988; 1: 25-31.

21 Bai TR. Abnormalities in airway smooth muscle in fatal asthma: a comparison between trachea and bronchus. Am J Respir Crit Care Med 1991; 143: 441-443.

22 Opazo Saez A, Seow C, Paré P. Peripheral airway smooth muscle mechanics in obstructive airways disease. Am J Respir Crit Care Med 2000; 161: 910-917.

23 Noble PB, Jones RL, Cairncross A, et al. Airway narrowing and bronchodilation to deep inspiration in bronchial segments from subjects with and without reported asthma. J Appl Physiol 2013; 114: 1460-1471.

24 Cerrina J, Le Roy Ladurie M, Labat C, et al. Comparison of human bronchial muscle responses to histamine in vivo with histamine and isoproterenol agonists in vitro. Am Rev Respir Dis 1986; 134: 57-61.

25 Goldie RG, Papadimitriou JM, Paterson JW, et al. Influence of the epithelium on responsiveness of guinea-pig isolated trachea to contractile and relaxant agonists. Br J Pharmacol 1986; 87: 5-14.

26 Mitzner W. Airway smooth muscle: the appendix of the lung. Am J Respir Crit Care Med 2004; 169: 787-790.

27 Sant'Ambrogio G, Widdicombe J. Reflexes from airway rapidly adapting receptors. Respir Physiol 2001; 125: 33-45.

28 Balzar S, Wenzel SE, Chu HW. Transbronchial biopsy as a tool to evaluate small airways in asthma. Eur Respir J 2002; 20: 254-259

29 Wang L, Chitano P, Paré PD, et al. Upregulation of smooth muscle Rho-kinase protein expression in human asthma. Eur Respir J 2020; 55: 1901785.

30 Lan B, Deng L, Donovan GM, et al. Force maintenance and myosin filament assembly regulated by Rho-kinase in airway smooth muscle. Am J Physiol Lung Cell Mol Physiol 2015; 308: L1-L10.

31 Yarova PL, Stewart AL, Sathish V, et al. Calcium-sensing receptor antagonists abrogate airway hyperresponsiveness and inflammation in allergic asthma. Sci Transl Med 2015; 7: $284 \mathrm{ra60}$

32 Flavahan NA, Aarhus LL, Rimele TJ, et al. Respiratory epithelium inhibits bronchial smooth muscle tone. J Appl Physiol 1985; 58: 834-838.

33 Gon Y, Hashimoto S. Role of airway epithelial barrier dysfunction in pathogenesis of asthma. Allergol Int 2018; 67: $12-17$.

34 Sparrow MP, Mitchell HW, Omari TI. The epithelial barrier and airway responsiveness. Can J Physiol Pharmacol 1995; 73: 180-190

35 Fredberg JJ, Jones KA, Nathan M, et al. Friction in airway smooth muscle: mechanism, latch, and implications in asthma. J Appl Physiol 1996; 81: 2703-2712.

36 Terracciano R, Pelaia G, Preianò M, et al. Asthma and COPD proteomics: current approaches and future directions. Proteomics Clin Appl 2015; 9: 203-220.

37 Vicente CT, Revez JA, Ferreira MAR. Lessons from ten years of genome-wide association studies of asthma. Clin Transl Immunology 2017; 6: e165.

38 Pascoe CD, Me O, Arsenault BA, et al. Gene expression analysis in asthma using a targeted multiplex array. BMC Pulm Med 2017; 17: 189. 
39 Rosner SR, Pascoe CD, Blankman E, et al. The actin regulator zyxin reinforces airway smooth muscle and accumulates in airways of fatal asthmatics. PLoS One 2017; 12: e0171728.

40 Beamish JA, He P, Kottke-Marchant K, et al. Molecular regulation of contractile smooth muscle cell phenotype: implications for vascular tissue engineering. Tissue Eng Part B Rev 2010; 16: 467-491.

41 Layne MD, Endege WO, Jain MK, et al. Aortic carboxypeptidase-like protein, a novel protein with discoidin and carboxypeptidase-like domains, is up-regulated during vascular smooth muscle cell differentiation. J Biol Chem 1998; 273: 15654-15660.

42 Turczyńska KM, Swärd K, Hien Tran T, et al. Regulation of smooth muscle dystrophin and synaptopodin 2 expression by actin polymerization and vascular injury. Arterioscler Thromb Vasc Biol 2015; 35: 1489-1497. 